\title{
Multiple Z-plasty for pilonidal sinus: How I do it
}

\author{
Atul K. Sharma, MBBS ${ }^{1}$, Rakesh K. Sharma, MS (General Surgery)', \\ Santosh K. Sharma, MSc (Med. Biochemistry) \\ 1 Resident Doctor, General Surgery, Government Medical College and A.G.H. Kota, India. \\ 2 Associate Professor, General Surgery, Government Medical College and A.G.H. Kota, India. \\ 3 Senior Demonstrator, Government Medical College and A.G.H. Kota, India.
}

Key words: Pilonidal Sinus; Z-plasty.

\section{Introduction}

Multiple Z-plasty is a novel recent technique for the treatment of symptomatic sacrococcygeal pilonidal disease. [1] A pilonidal sinus is a surgical conditions which, though appearing trivial to the patient, involves a radical surgical procedure entailing a considerable stay in hospital, discomfort and loss of earnings $[2,3]$. It is responsible for much morbidity and its treatment places a burden on hospital and community resources.

This technique was first reported in the literature in 2006 patronizing its superiority over z-plasty and other techniques for the treatment for pilonidal sinus [1]. Sharma [1], concluded that it avoids a midline scar; it makes the natal cleft shallow, thereby eliminating the suction effect and reducing sweat accumulation; it is easy to learn, safe and effective.

\section{Principles in Multiple Z-plasty}

1. The principle of the treatment of pilonidal sinus by excision and multiple Z-plasty reconstruction acknowledges contemporary concepts of the aetiology of this condition and seeks to eliminate these causal factors, thus making recurrence less likely.

2. Multiple Z-plasties excise the inflamed area as well as convert the deep natal cleft into a plateau.

3. Multiple Z-plasty requires less lateral undermining, thus it can be easily accomplished and also has less blood loss, no necrosis of the

Correspondence: Atul Kumar Sharma, Midil School Street, Deeg (Bharatpur) Raj. Pin-321203 India

Email: docatul83@gmail.com

The Sri Lanka Journal of Surgery 2012; 30(1):26-29. apices of the flaps due to smaller flap size and less numbness.

\section{Surgical Technique}

In this procedure patients are given spinal anaesthesia after taking consent and are placed prone in the jack knife position. (Fig.1)

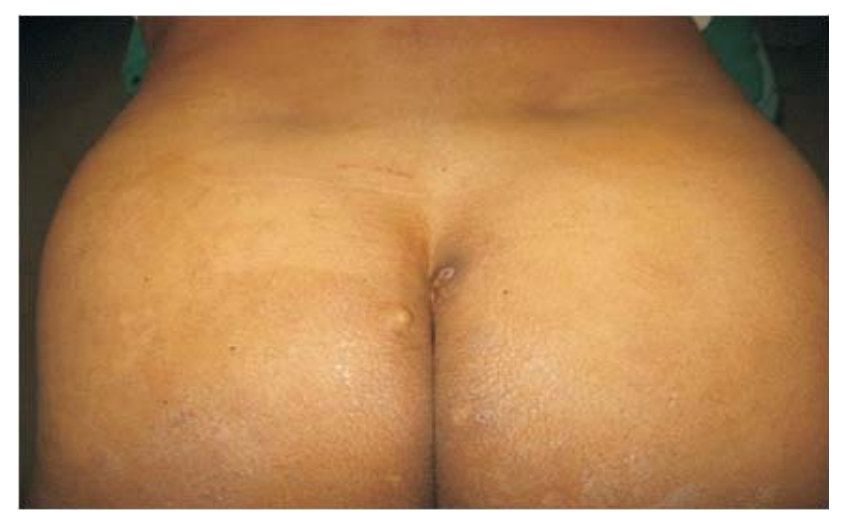

Figure 1. Patient lying prone (Jack Knife Position)

The buttocks are strapped with sticking plaster to keep them apart for better exposure of the operative area. (Fig.2)

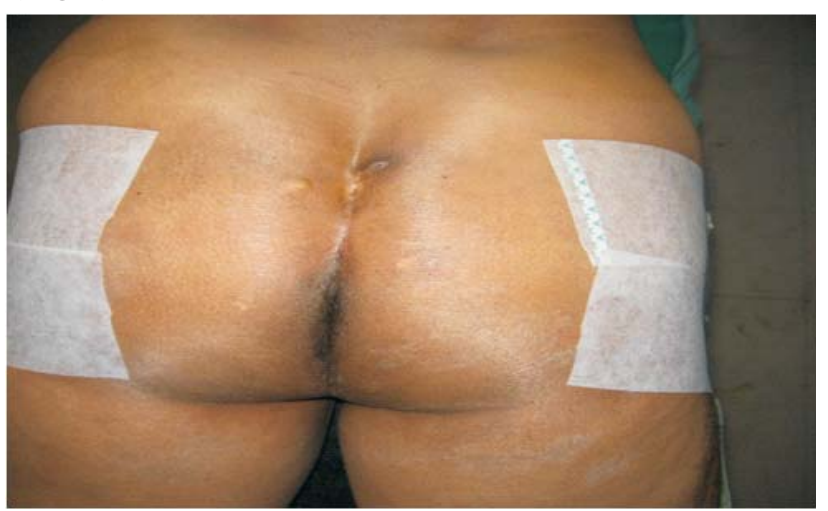

Figure 2. The patient's buttocks are separated by adhesive plaster 
After aseptic painting using 10\% povidone iodine solution and draping the area, the sinus is probed to dilate its opening. (Fig 3)

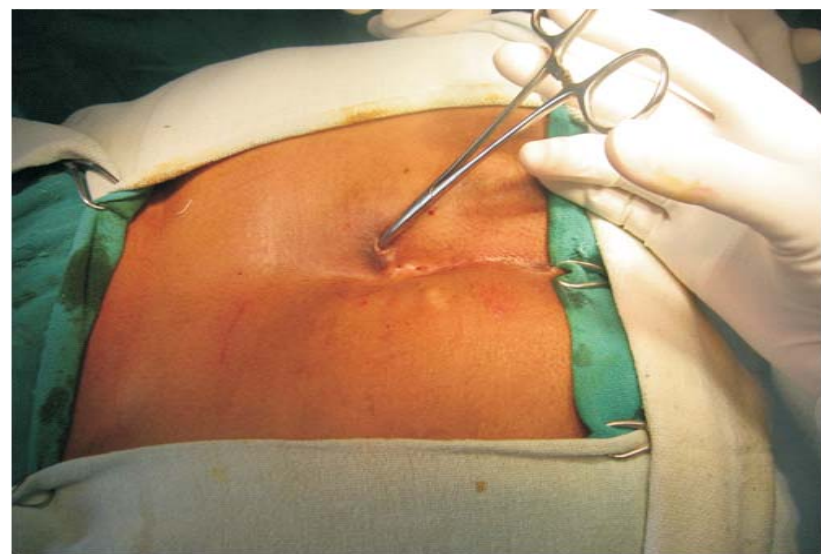

Figure 3. Probing the sinus after aseptic painting and draping

A 1:200,000 adrenaline in saline solution is infiltrated in the tissue around the sinus tract to minimize oozing during excision of the tract. (Fig 4)

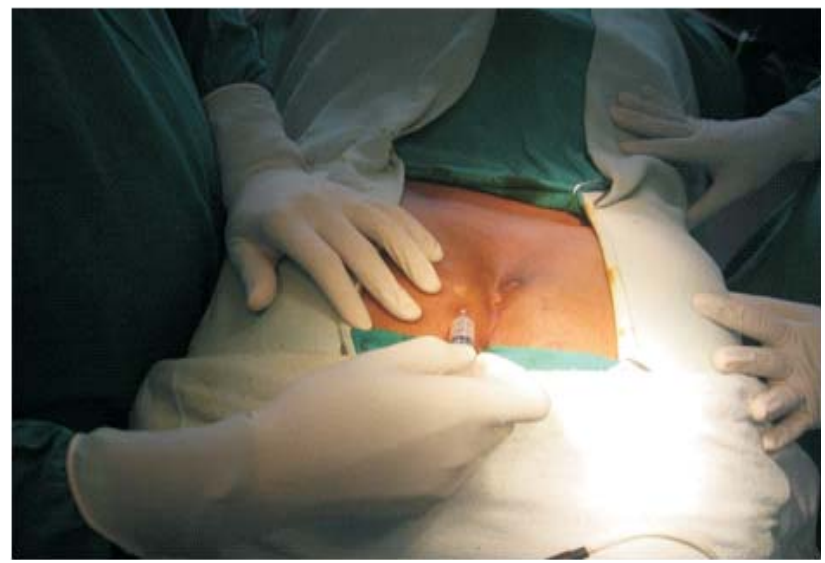

Figure 4. Injecting Saline and adrenaline $(1: 200,000)$ in the tissue around the tract

An adequate volume of methylene blue ( 2 to $3 \mathrm{ml}$ ) plus a few drops of hydrogen peroxide are injected through the sinus opening to delineate the complete sinus tract and its branches if any. (Fig 5)

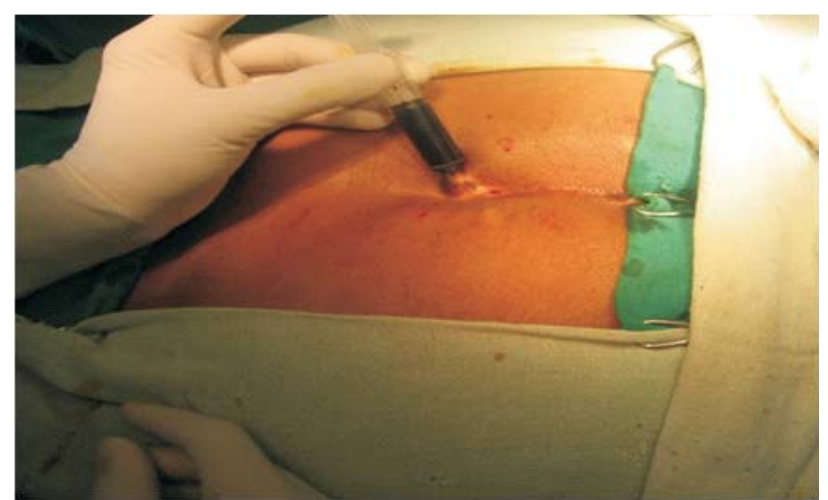

Figure 5. Injecting methylene blue into the sinus tract
The complete sinus tract is excised using an elliptical incision encircling the sinus opening. (Fig 6)
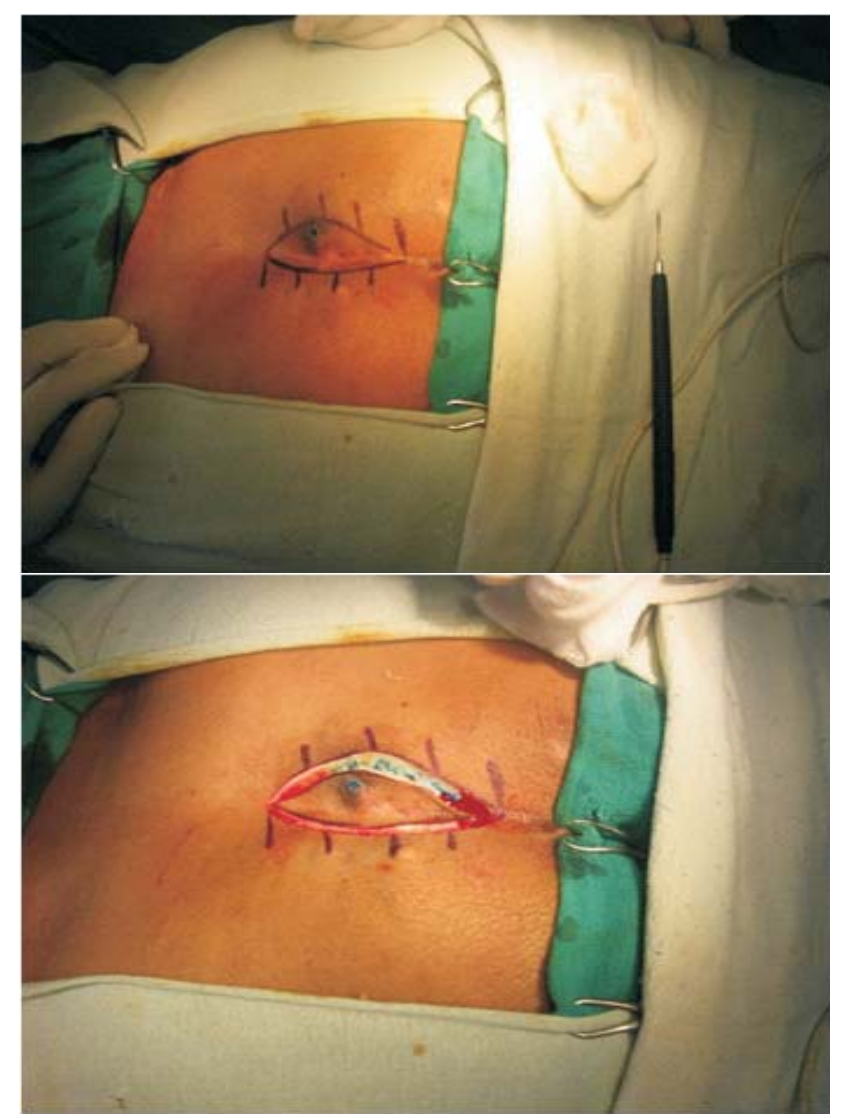

Figure 6. Encircling sinus tract with elliptical incision

The sinus opening along with all congenital pits is encircled with an elliptical incision and the tract is cored out completely taking care not to enter the tract at any time. (Fig 7A\& 7B)

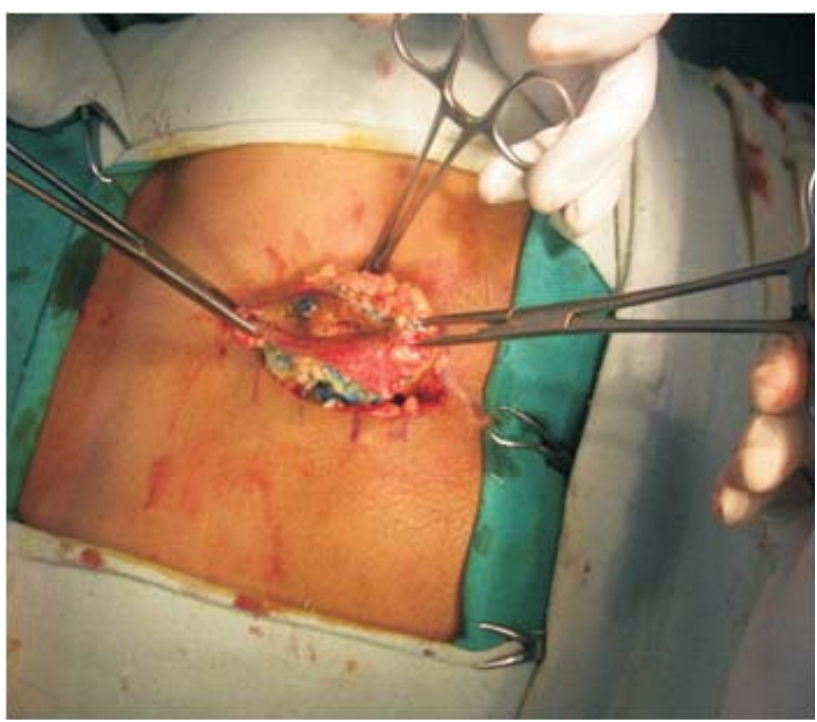

Figure 7A. Coring out the entire sinus tract 


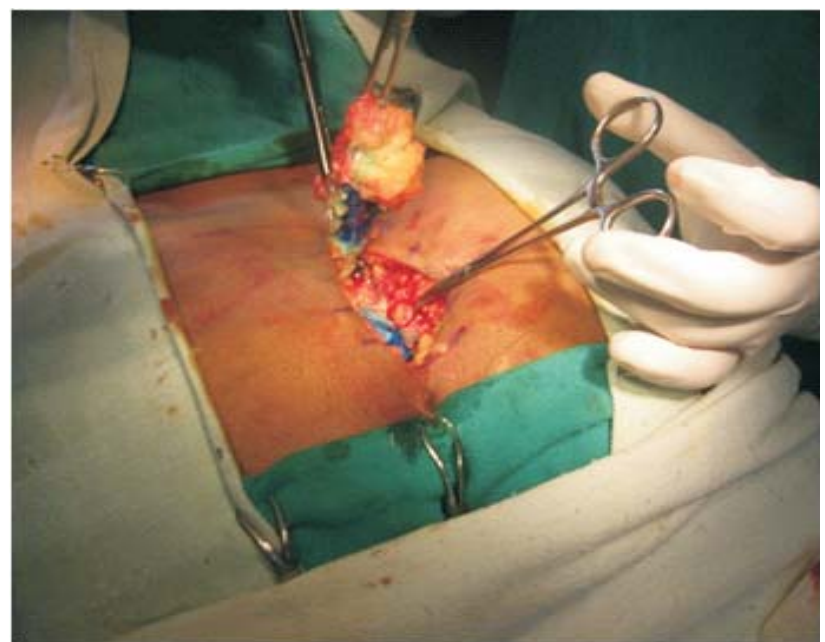

Figure 7B. Coring out the entire sinus tract

Haemostasis is achieved by unipolar cautery. Multiple Z-plasty is carried out by making multiple oblique incisions across the main wound in full depth. (Fig 8A \& 8B)

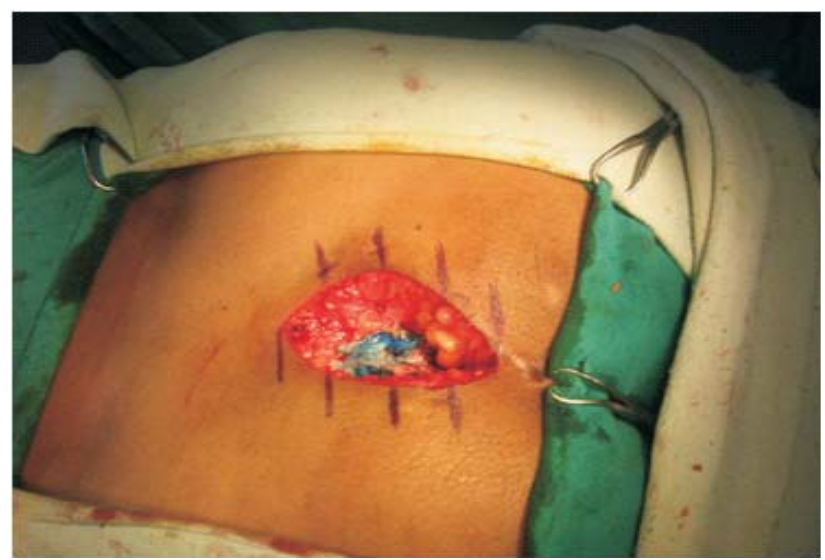

Figure 8A. Wound after excision of sinus tract with marking for multiple Z-plasty

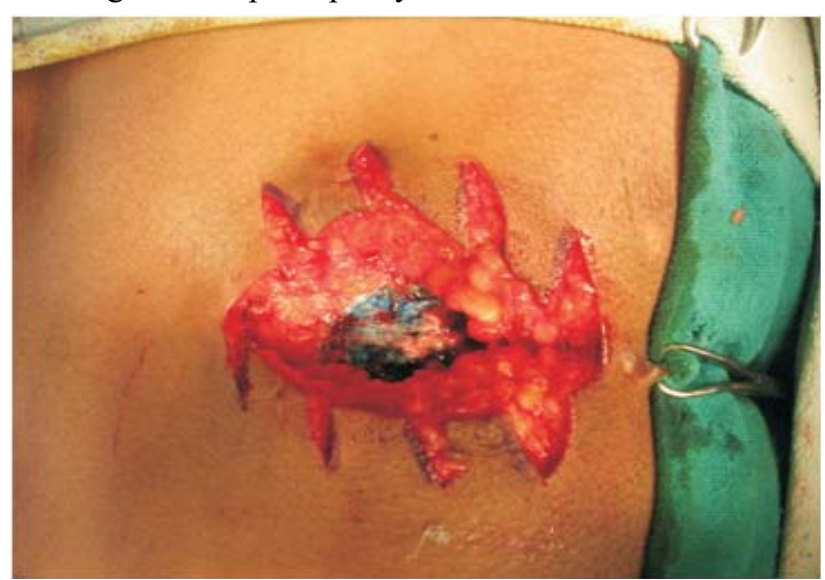

Figure 8B. Making flaps for multiple Z-plasty
A closed suction drain of 18 Fr size is left in the wound and is taken out through a separate stab wound (Fig 9) and fixed to the skin using 3-0 nylon.

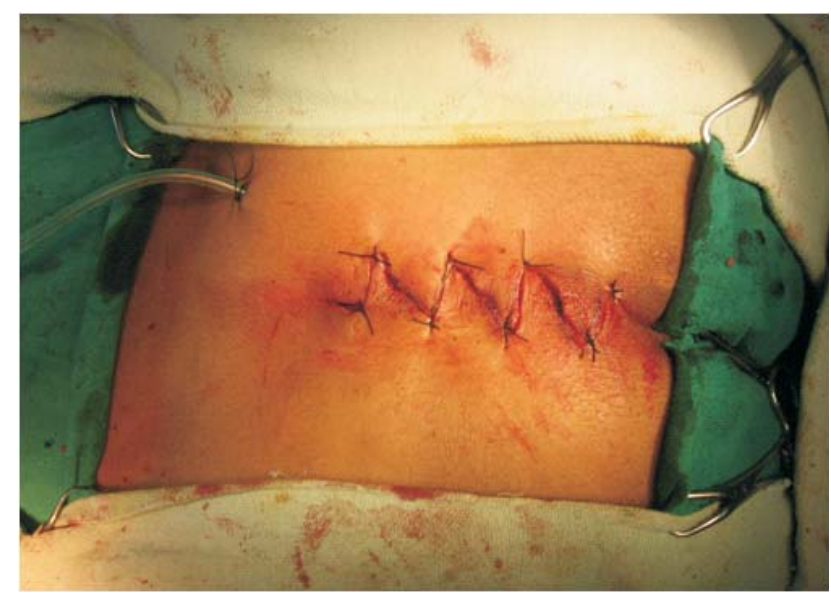

Figure 9. Putting closed suction drain and applying holding sutures for interdigitating the flaps

These flaps are suitably interdigitated and sutured in one layer with nylon 3-0 interrupted sutures. (Fig 10A)

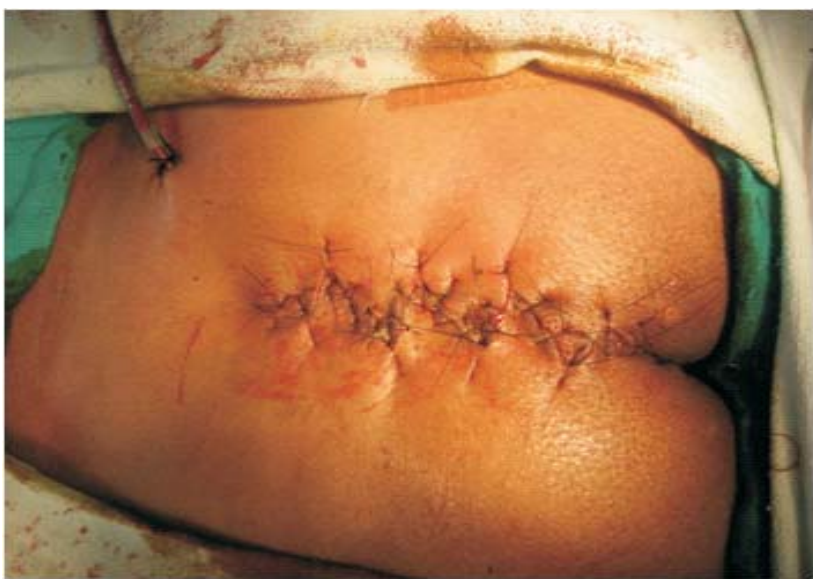

Figure 10A. Suturing the wound after interdigitating the flaps

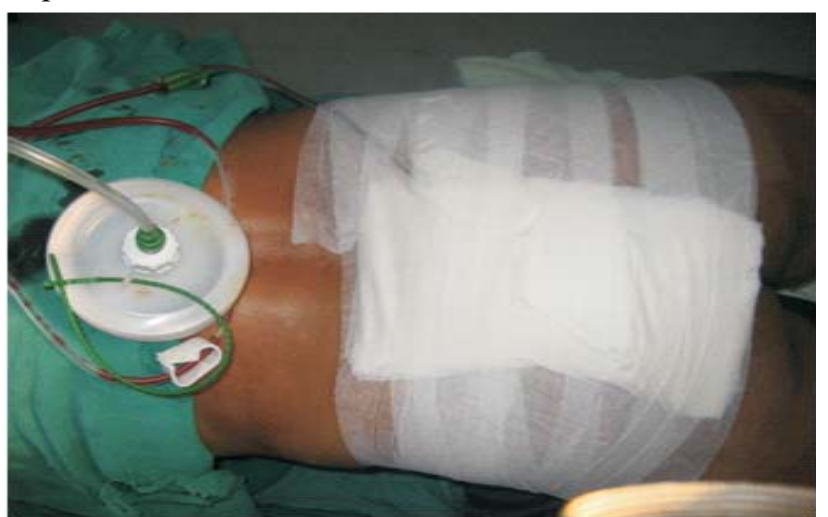

Figure 10B. Applying the firm pressure dressing using adhesive plaster 
The wound is dressed after applying povidone iodine ointment over the suture line using sterile cotton pads, and firm pressure is used while applying the adhesive plaster to keep the cotton dressing pads in position (Fig.10 B).

The patient is kept in bed in the prone or lateral position for 5 days until the wound gets adequate tensile strength. The suction drain is removed on the 3 rd to 5 th post operative day depending on the cessation of drainage of fluid through it.

Alternate sutures are removed on the 5 th postoperative day and the patient is usually discharged allowing routine activities and being instructed to avoid squatting for defaecation until two weeks postoperatively to avoid traction on the suture line.

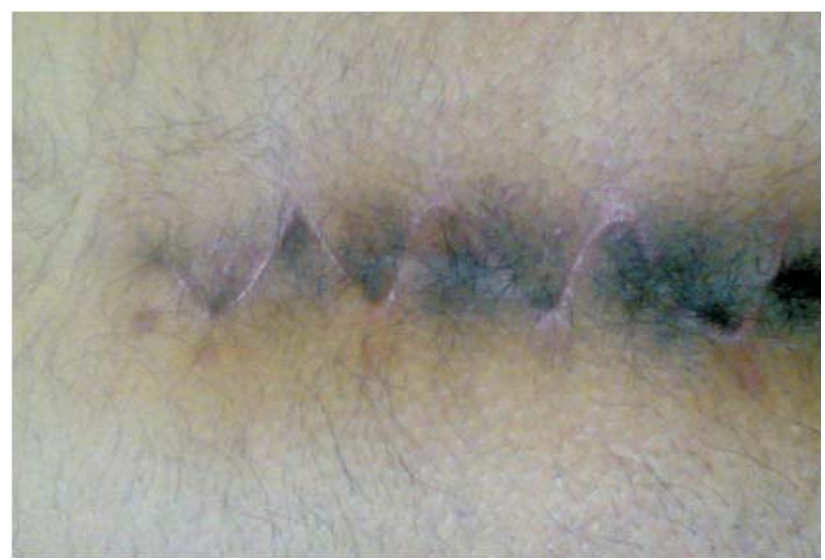

Figure 11. Completely healed sinus after suture removal

The patient is discharged on the 5th day if there is no other indication to keep the patient hospitalized. The remaining sutures are removed on the 8th -10 th day. (Fig.11)

\section{Discussion}

Pilonidal disease is a benign condition and patients apparently outgrow its infective complications. It is therefore imperative that the treatment is not worse than the disease itself. It is recommended the midline pits are excised with lateral open drainage of any associated abscess [4].

The ideal procedure for this condition would be an operation that:

- $\quad$ is in sequence simple to perform

- requires short hospitalization

- allows a rapid return to normal activity [5]
Multiple Z-plasty has surpassed other surgical modalities for the treatment of pilonidal sinus in terms of morbidity, healing time, complications and recurrence [1]. It has the following advantages over other techniques:

1. Less healing time and less post operative hospital stay [1].

2. No local hematoma, wound infection or wound dehiscence with minimal recurrence.

3. Technically less demanding.

4. Avoids a midline scar.

5. Makes the natal cleft shallow, thereby eliminating the suction effect and reducing sweat accumulation.

6. Easy to learn, safe, and effective.

However, newer, less invasive techniques have been introduced, like vacuum assisted closure of wounds [6] and platelet rich plasma concentrate infiltration in the wound [7]. However, these are in their experimental phase, and further long term studies are required to evaluate their efficacy in the treatment of pilonidal disease.

\section{References}

1. Pradeep Purushottam Sharma. Multiple Z-Plasty in Pilonidal Sinus A New Technique under Local Anesthesia. World J Surg (2006) 30: 2261-2265

2. Coates C,Wendy,Rosen's Emergency Medicine concept snd clinical practice fifth edition.Mosby 2002;1350-1351.

3. Karydakis GE. New approaches to the problem of pilonidal sinus. Lancet 1973;2:1414-15

4. Arugmugan PJ, Chanrase Karan TV, Morgan AR. the rhomboid flap for pilonidal disease.Colorectal Dis 2003;87:567-72

5. Hull TL, Wu James. Pilonidal disease. Surg Clin North Am 2002;82:1169-1185.

6. McGuinness JG, Winter DC, O'Connell PR. Vacuum assisted closure of a complex pilonidal sinus. Dis Colon Rectum 2003;46(2):274-276

7. Spyridakis M, Christodoulidis G, Chatzitheofilou C, Symeonidis D, Tepetes K. The role of the platelet-rich plasma in accelerating the wound-healing process and recovery in patients being operated for pilonidal sinus disease: preliminary results. World J Surg 2009;33(8):1764-1769 